\title{
Persistencia de las ideas de Lenz en el estudio del contacto mapudungun-español. Algunos ejemplos de la investigación actual
}

\author{
Felipe Hasler ${ }^{1}$ \\ Universidad de Chile, Chile \\ Aldo Olate ${ }^{2}$ \\ Universidad de la Frontera, Chile \\ Guillermo Soto ${ }^{3}$ \\ Universidad de Chile, Chile
}

\begin{abstract}
Resumen
En el presente trabajo, se examinan fenómenos fonológicos, morfosintácticos y pragmáticos que permiten sostener la persistencia
\end{abstract}

1 Para correspondencia, dirigirse a: Felipe Hasler (keche.felipe@gmail.com), Universidad de Chile, Facultad de Filosofía y Humanidades, Departamento de Lingüística. Av. Capitán Ignacio Carrera Pinto 1025, Ñuñoa, Santiago, Chile.

2 Para correspondencia, dirigirse a: Aldo Olate (aldo.olate@ufro.cl), Universidad de La Frontera, Facultad de Educación, Cs. Sociales y Humanidades, Departamento de Lengua, Literatura y Comunicación. Av. Francisco Salazar 01145, Temuco, Chile.

3 Para correspondencia, dirigirse a: Guillermo Soto (gsoto@uchile.cl), Universidad de Chile, Facultad de Filosofía y Humanidades, Departamento de Lingüística. Av. Capitán Ignacio Carrera Pinto 1025, Ñuñoa, Santiago, Chile. 
de las ideas de Rodolfo Lenz (1863-1938) en la lingüística de contacto actual. Tras años de cierto olvido o minusvaloración en la lingüística hispánica, tendencias recientes de la contactología permiten apreciar a un Lenz visionario que, anticipándose a su época y contra el clima ideológico vigente en el entorno hispanoamericano, sentó las bases del estudio científico del español en contacto con el mapudungun y, más ampliamente, en contacto con lenguas indígenas.

Palabras clave: Rodolfo Lenz, mapudungun, español, contacto mapudungun-español, contactología, lingüística de contacto.

\title{
The Persistence of Lenz ideas in Mapudungun-Spanish
} CONTACT STUDIES. SOME EXAMPLES FROM CURRENT RESEARCH

\begin{abstract}
In the present work, phonological, morphosyntactic and pragmatic phenomena are examined that allow us to sustain the persistence of the ideas of Rodolfo Lenz (1863-1938) in current contact linguistics. After years of some neglect or undervaluation in Hispanic linguistics, recent trends in contactology allow us to appreciate a visionary Lenz who, anticipating his time and against the current ideological climate in the Spanish-American environment, laid the foundations for the scientific study of Spanish in contact with Mapudungun and, more broadly, in contact with indigenous languages.
\end{abstract}

Keywords: Rodolfo Lenz, Mapudungun, Spanish, MapudungunSpanish contact, contactology, contact linguistics.

Recibido: $13 / 05 / 19 \quad$ Aceptado: 04/06/20

\section{INTRODUCCIÓN ${ }^{4}$}

A fines del siglo XIX, un todavía joven Rodolfo Lenz publica en alemán sus Estudios chilenos I-VII, que recién medio siglo más tarde, en 1940, serán

4 El presente capítulo se inscribe en el proyecto Fondecyt Regular 1180071 "TAM, evidencialidad, fenómenos asociados a la valencia verbal y la codificación de eventos complejos en el castellano de contacto hablado por bilingües mapuche/castellano. Un estudio comparativo del sistema verbal" de CONICYT. 
traducidos al español por Amado Alonso y Raimundo Lida en la Biblioteca de Dialectología Hispanoamericana de Buenos Aires. A juicio de Rodolfo Oroz, estos estudios entregan "la primera descripción rigurosamente científica de la pronunciación chilena" (1962: 28); y más aun, en el decir de Alonso, constituyen "servicios excepcionales" prestados por Lenz a la lingüística hispánica (1940a: 277). En ellos, el lingüista alemán, bajo el supuesto de que el español habría evolucionado "probablemente en Chile más que en ninguna otra región de la tierra" (Lenz 1940: 87), se dedica a caracterizar las peculiaridades fonéticas del español coloquial hablado en Chile, poniendo como telón de fondo un español general que, según Alonso (1940b), Lenz, por su formación en Alemania, desconocía. Si bien sus caracterizaciones de fenómenos específicos del español de Chile, como, entre otros, la palatalización de las consonantes velares ante vocal anterior o el rehilamiento de la /r/, siguen a firme, la hipótesis que propuso para explicar las diferencias entre el español hablado en Chile y el general, esto es, la idea de que "el español de Chile (es decir, la pronunciación del pueblo bajo) es, principalmente, español con sonidos araucanos" (Lenz 1940: 249), no corrió la misma suerte y, tras la crítica que de ella hiciera Alonso, la opinión dominante en la lingüística hispanoamericana ha sido que Lenz se equivocaba tanto al atribuir a esta variedad una serie de peculiaridades fonéticas que la distanciarían grandemente del español general, como al explicar varios de estos fenómenos atribuyéndolos a un sustrato o adstrato mapuche. Más ampliamente, el proyecto fundamental de Lenz - consistente, como ha destacado Sánchez, en "comprobar [la influencia de la lengua mapuche] en el castellano hablado por el "bajo pueblo chileno"" (1992: 278) - no fue proseguido mayormente y ciertamente no tuvo, en los lingüistas que vinieron tras él, la perspectiva comprehensiva con que el lingüista alemán lo llevó a cabo, bajo el entendido de que con esta tarea, que llamaba filológica, contribuía a una caracterización social y psicológica del pueblo chileno, que entendía se había ido conformando en una situación de contacto no solo lingüístico, sino vital entre españoles y mapuches. Baste una cita para entender la relevancia sociocultural que, en el contexto chileno y precisamente para una mejor comprensión de lo chileno, daba Lenz al estudio del contacto:

Pues si, en último término, Chile debe lo que es a su pueblo bajo, a esa raza de sangre mezclada española y araucana, no parecerá ya un asunto de poca importancia el indagar las especialidades del lenguaje del huaso chileno. (Lenz 1894: 132)

Esta relevancia, que no resulta extraña considerando el contexto académico europeo de la época, no debiera hacernos pasar por alto la importancia 
particularmente lingüística que advierte Lenz en esta materia, en tanto, a partir de la información de que disponía tanto por su formación lingüística en Alemania como por su experiencia en Chile, se daban en este último país excelentes condiciones para estudiar el cambio lingüístico en situación de contacto y, consecuentemente, el modo en que las leyes de evolución lingüística operan en el contexto de un mestizaje extendido que va aparejado a la extensión de la lengua del poder conquistador. ++++++

Lenz esboza la hipótesis sustratista en más de una obra y respecto de diversos rasgos del español de Chile. De los fenómenos fonéticos para los que propone esta causa en sus Estudios chilenos, probablemente el más conocido sea la tesis del origen mapuche de la realización asibilada del grupo consonántico/tr/, común en el español de Chile. En contraposición a la tesis de Lenz, Alonso (1925) muestra que este fenómeno no es exclusivamente americano, sino que se da también en la península, lo que sugeriría causación interna, o incluso influencia española norteña (Lapesa 1991, 1992). En la actualidad se reconoce que la realización asibilada de este grupo se asocia más ampliamente a la asibilación de / $\mathrm{r} / \mathrm{y}$ no es exclusiva de Chile en América (Canfield 1988 [1981]; Lapesa 1991, 1992; Fontanella de Weinberg 1992; Penny 2000). Con un alcance mayor, y en el mismo libro en que publica la traducción de los Estudios chilenos, el lingüista español escribe una crítica rotunda y de hondo impacto contra el sustratismo de Lenz, llegando a afirmar que "sin excepción alguna, es inadmisible la atribución del araucanismo para cada uno de los fonemas en que Lenz lo creyó ver" (Alonso 1940b: 288). A partir de la crítica de Alonso, con unas pocas excepciones la posición ideológica dominante en la dialectología hispanoamericana ha sido contraria a la tesis sustratista. En palabras de Fontanella de Weinberg: "Como conclusión, podemos afirmar que a esta altura de los estudios del español americano resulta injustificable ya atribuir en términos generales sus peculiaridades al influjo del sustrato" (1992: 31; en el mismo sentido, Lope Blanch 1992 y Sánchez Méndez 2003).

No obstante lo anterior, transcurridos ya más de cien años de los Estudios chilenos, la idea de que el contacto entre español y mapudungun es relevante para una mejor comprensión de ambas lenguas (planteamiento con frecuencia pasado por alto en esta discusión) y que la influencia del mapudungun en el español hablado en Chile pudo no limitarse al léxico merece una revisión que vaya más allá de la cuestión general de la crítica al sustratismo como explicación del origen del español de América, una refutación que, por lo demás, como sugiere Bernaschina (2013), parece haber respondido en su momento no solo a razones científicas, sino también ideológicas, en el marco, según este autor, "de una fase de consolidación de la filología hispánica en la que Menéndez Pidal, Alonso y Castro 
trabajaron conjuntamente, persiguiendo objetivos pan-hispánicos similares" (Bernaschina 2013: 120) que chocaban con el proyecto filológico de Lenz, que destacaba la diversidad. En efecto, el importante desarrollo que desde fines del siglo pasado ha venido teniendo el estudio del contacto entre el español y las lenguas indígenas, y que obedece tanto a "innovaciones teóricas y metodológicas en el campo de la contactología y de la lingüística" como a cambios socioculturales e ideológicos en la región (Soto y Hasler 2011; Olate, Becerra y Alonqueo 2011), favorece una relectura del aporte de Lenz que rescate sus aspectos más propiamente lingüísticos, en particular en lo que respecta a la lingüística de contacto. Más que resucitar una discusión específica, la del sustrato, en los términos científicos e ideológicos en que esta se planteó en su tiempo, nos interesa relevar la persistencia de ciertas ideas de Lenz sobre el contacto mapudungun-español, a partir del examen de diversos estudios contemporáneos que, en mayor o menor grado, pueden entenderse como relacionados con ellas. Como ya hemos adelantado, nuevas visiones de la contactología, que ponen en cuestión la división simple entre cambios lingüísticos motivados interna y externamente, el creciente interés por el estudio de las lenguas y culturas americanas en el contexto actual de reetnificación y demandas políticas y sociales de los pueblos indígenas, así como los datos genéticos sobre población chilena, que vienen a confirmar la hipótesis del mestizaje, central en la construcción del argumento de Lenz, permiten ver hoy en él a un adelantado de los actuales estudios de contacto lingüístico en América.

En lo que sigue, expondremos sintéticamente investigaciones sobre el contacto español-mapudungun que cubren desde el nivel fonéticofonológico hasta el gramatical-discursivo, pasando por el morfosintáctico. Estos estudios, claramente focalizados en la llamada lingüística interna, buscan una mejor comprensión de propiedades del español de Chile, del mapudungun y del español hablado por mapuches, o español mapuchizado. Tomados en conjunto, permiten apreciar que el proyecto científico de Lenz -que va más allá de una hipótesis específica sobre una variedad del español americano-, lejos de haber sido refutado, establece las bases sobre las que se construyen los actuales estudios de la lingüística de contacto españolmapudungun. Al observar la investigación desarrollada por Lenz en el campo del contacto lingüístico, puede apreciarse que tanto sus intuiciones y conceptualizaciones como las metodologías que desarrolló forman parte de las que actualmente, mutatis mutandis, se emplean en la gran mayoría de los estudios de lingüística de contacto. Su aguda conciencia científica lo llevó a proponer la necesidad del conocimiento descriptivo de las gramáticas de los dos códigos convergentes para sustentar su intuición sobre el español de Chile (Lenz 1895-1897), idea que encontramos desarrollada actualmente en 
el principio de distancia tipológica en situaciones de contacto (Thomason y Kaufman 1988; Thomason 2001, 2013; Heine y Kuteva 2005; Aikhenvald y Dixon 2007; Matras 2009, 2013; Siemund y Kitana 2008, entre otros). En esta misma línea, la conciencia tipológico-descriptivista de Lenz, a la hora de estudiar la lengua indígena, es cercana a las desarrolladas por los descriptivistas norteamericanos de 1920-1950 (Sapir 1921), pues promueve la descripción de la lengua indígena desde cánones distintos a los tradicionales, que basaban el ejercicio descriptivo en procedimientos escolásticos y latinos (Lenz 1895-1897). El alcance de sus propuestas muestra que las intuiciones respecto de las lenguas estaban muy en línea con posiciones que actualmente están teórica y empíricamente validadas. Lo que encontramos en Lenz son las primeras ideas para estudiar procesos de cambio en el español de América y su interacción con lenguas indoamericanas (Palacios 2011; Olate, Becerra y Alonqueo 2011; Hasler 2012, entre otros). En otras palabras, las propuestas de Lenz pueden considerarse fundamentales para (1) entender el fenómeno del contacto como agente promotor del cambio y (2) reafirmar que existen dominios lingüísticos (y cognitivos) más susceptibles al cambio lingüístico por contacto, situación que en el español de América se asocia a la incidencia de las lenguas indígenas, con un sentido de sustrato vivo (Zimmermann 2001).

\section{FONOLOGÍA}

\subsection{HISPANISMOS LÉXICOS Y SU RELEVANCIA PARA EL ESTUDIO DEL CONTACTO EN EL PERÍODO COLONIAL}

Siguiendo la idea de Lenz (1895-1897 y 1940) en el sentido de que la investigación del contacto entre español y mapudungun podría ayudar al conocimiento de la estructura lingüística de ambas lenguas en el pasado, Hasler y Soto (2012) han planteado que el estudio de los hispanismos léxicos registrados en el período colonial (ss. XVI y XVII) por Febrés en su Arte de la lengua general del Reyno de Chile (1765) permite proponer reconstrucciones de los sistemas fonológicos de las lenguas en contacto en aquella época. Específicamente, el examen del modo en que se pronunciaban en mapudungun y se escribían por Febrés préstamos del español, como lachu, por lazo, contribuye a proponer la existencia, en la época considerada, de ciertas consonantes en una y otra lengua. En particular, préstamos de palabras españolas en que figuran cuatro fenómenos muy estudiados en la tradición de 
la lingüística histórica de esta lengua, a saber: el seseo, el surgimiento de la $/ \mathrm{x} /$, la pérdida de la $/ \mathrm{s} /$ implosiva y la distinción entre $/ \mathrm{b} /-/ \mathrm{v} /{ }^{5}$, muestran una serie de correspondencias de fonemas, fonos y grafemas entre el mapudungun y el español en el período estudiado por Febrés.

En primer lugar, los fonemas $/ \mathrm{s} / \mathrm{y} / \Theta /$ del español, esto es, las consonantes sordas dental ( $\underline{\text { señora }}$ ) e interdental (lazo), se corresponden, en mapudungun, ya con el fonema africado sordo $/ \mathrm{t} /$ en dialectos nortinos (escrito como $<\mathrm{ch}>$, por ejemplo en lach $u<$ esp. lazo), ya con la fricativa interdental/Ө/, en sureños (escrito con $<\mathrm{s}>$, como en mans $u<$ esp. manso). En segundo lugar, con respecto al surgimiento del fonema $/ \mathrm{x} /$ (consonante fricativa velar sorda), es posible plantear que el español de la época colonial chilena no tenía aún este fonema, cuya realización actual responde al proceso más amplio de reajuste de las sibilantes que caracteriza el paso del español medieval al moderno. Esto significa que probablemente se conservaba el fonema $/ \mathrm{J} /$, o /3/ (consonantes fricativas palatales, sorda la primera y sonora la segunda), pues en palabras que los contenían, estos fonemas se correspondían, en mapudungun, con el fonema $/ \mathfrak{t} /$, como en el caso del español aguja que pasa al mapudungun como acucha. En contraste con los préstamos registrados por Febrés, en los estudios de hispanismos léxicos en el mapudungun del siglo XIX, como el de Giese (1947-1949), es posible observar que en préstamos modernos, la / $\mathrm{x} /$ del español se asimila a la $/ \mathrm{k} /$ del mapudungun, como en el caso del español espejo, que pasa al mapudungun como espeko. Por su parte, la /s/ implosiva del español de la época se corresponde con la consonante retrofleja / / $/$ del mapudungun, escrita como $<\mathrm{r}>$ (irpada $<$ esp. espada). Finalmente, con respecto a la distinción entre $/ \mathrm{b} / \mathrm{y} / \mathrm{v}$, Lenz señala que en ocasiones Febrés las representaba a través de la grafía $\langle\mathrm{v}\rangle$, la mayoría de las veces se reproducían por la grafía $<\mathrm{hu}>$, correspondiente al fonema $/ \mathrm{w} /$, como en cahuello $<$ esp. caballo y, en ocasiones, por $<$ p $>$, correspondiente al fonema /p/, como napur < esp. nabo. El Cuadro 1 sintetiza las relaciones expuestas:

\footnotetext{
5 El seseo refiere a la pérdida de la distinción entre los fonemas /s/ (fricativo, dental, sordo) y/ $\Theta /$ (fricativo, interdental, sordo), hoy común a todo el español americano, pero no al peninsular; el surgimiento de la /x/, por su parte, dice relación con el fonema fricativo, velar y sordo, propio del español moderno; la pérdida de /s/ implosiva, por otro lado, refiere a la elisión o aspiración de /s/ en posición posvocálica, esto es, después del núcleo de la sílaba; y la distinción $/ \mathrm{b} /-/ \mathrm{v} /$, finalmente, al contraste fonológico entre una consonante bilabial y otra labiodental, inexistente hoy en el español general.
} 


\begin{tabular}{|c|c|c|c|}
\hline $\begin{array}{c}\text { Fonemas en } \\
\text { español }\end{array}$ & $\begin{array}{l}\text { Correspondencia con fo- } \\
\text { nemas en mapudungun }\end{array}$ & Grafemas & Ejemplos \\
\hline \multirow[t]{2}{*}{$/ \mathrm{s} /-/ \theta /$} & $\begin{array}{l}/ \mathrm{tg} / \\
\text { (dialectos nortinos) }\end{array}$ & $<\mathrm{ch}>$ & \multirow{2}{*}{$\begin{array}{l}\text { Lazu, lachu, de } \\
\text { lazo; } \\
\text { Mansu, manchu, } \\
\text { de manso; Chiñu- } \\
\text { ra, de señora }\end{array}$} \\
\hline & $\begin{array}{l}\text { / } \Theta / \text { (dialectos centrales } \\
\text { y sureños) }\end{array}$ & $\begin{array}{l}<\mathrm{ch}>,<_{\mathrm{S}}> \\
<\mathrm{z}>\end{array}$ & \\
\hline$|s /-| 3 \mid$ & $|\mathrm{t}|$ & $<\mathrm{ch}>$ & $\begin{array}{l}\text { Acucha, de aguja; } \\
\text { Achur, de ajos }\end{array}$ \\
\hline $\begin{array}{l}/ \mathrm{X} /(\mathrm{s} . \mathrm{XIX} \mathrm{y} \\
\mathrm{XX})\end{array}$ & $/ \mathrm{k} /$ & $<\mathrm{k}>$ & $\begin{array}{l}\text { Espeko, de espejo; } \\
\text { Sortika, de sortija }\end{array}$ \\
\hline /s/ implosiva & / & $<\mathrm{r}>$ & $\begin{array}{l}\text { Irpada, de espada; } \\
\text { Irtipu, de estribo }\end{array}$ \\
\hline$/ \mathrm{b} /-/ \mathrm{v} /$ & $/ \mathrm{v} /, / \mathrm{w} /, / \mathrm{p} /$ & $\begin{array}{l}<\mathrm{v}>,<\mathrm{hu}> \\
<\mathrm{p}>\end{array}$ & $\begin{array}{l}\text { Alvis, de alberjas; } \\
\text { Huaca, de vaca; } \\
\text { Napur, de nabo }\end{array}$ \\
\hline
\end{tabular}

Cuadro 1: Correspondencias entre fonemas del español y el mapudungun a partir de los hispanismos léxicos registrados por Febrés.

Considerando las correspondencias encontradas en Febrés, Hasler y Soto proponen que, en el período considerado por el jesuita catalán, el español en contacto aún no había experimentado el reajuste fonológico de las sibilantes medievales (Oroz 1962), pues esta lengua aún no presentaba el fonema $/ \mathrm{x} /$. Además, los autores plantean que el español en contacto conservaba la /s/ implosiva, lo que explica su realización en mapudungun como $/ \mathrm{t}$, sonido más próximo del sistema del mapudungun al sonido en cuestión. Finalmente, dada la existencia de dos soluciones alternativas para las labiales sonoras: una oclusiva bilabial sorda (distante solo en sonoridad de la realización prototípica de $/ \mathrm{b} /$ ) y otra aproximante velar, labializada sonora (bastante próxima a la realización típica de /v/), consideramos que es posible proponer que, al menos en un primer momento y entre los españoles que estaban en contacto con los mapuches, el español de la época colonial distinguía entre $/ \mathrm{b} / \mathrm{y} / \mathrm{v} /$.

Por otro lado, con respecto al mapudungun de la época, siguiendo esta línea argumental, es posible proponer que la lengua mapuche de este período 
tenía al menos dos variedades dialectales diferenciadas por la presencia o ausencia de $/ \Theta /$ : aquellos dialectos que contaban con $/ \Theta /$, en que los fonemas $/ \mathrm{s} /-/ \Theta /$ se realizaban a través de $/ \Theta /$, y aquellos que no contaban con este fonema, que pasaron a realizar estos fonemas del español con $/ \mathrm{t} /$.

\section{MORFOSINTAXIS}

\subsection{El SINTAGMA NOMINAL}

Tal como lo señala Lenz, lo importante para el estudio del castellano en contacto con la lengua mapuche radica en conocer el funcionamiento de ambos sistemas. De este proceso comparativo surge lo que se reconoce actualmente como la distancia tipológica entre las lenguas en contacto (Siemund y Kitana 2008; Thomason 2013). La distancia tipológica puede entenderse como aquellos dominios lingüístico-funcionales en que los códigos se diferencian mayormente en función de la sobrecodificación o subcodificación de elementos de un subdominio u otro. En el plano comparativo, a nivel de la estructura de la frase nominal, el mapudungun y el castellano presentan diferencias de tipo formal (Olate, Becerra y Alonqueo 2011; Olate, Pineda, Hasler y Soto, 2019).

En cuanto a las diferencias ligadas a la forma, el sintagma nominal mapuche no requiere establecer concordancia entre sustantivo, determinantes, adjetivos, ni algún otro modificador nominal a diferencia del español, cuyo sistema hiperconcordante está bien descrito. El mapuche tiende a especificar género y número solo en algunos casos, y mediante procedimientos no siempre concurrentes (como la anteposición de domo 'mujer/hembra' y wentru 'hombre/macho' al sustantivo en cuestión en el caso del género o la anteposición de la partícula $p u$ al sustantivo, como en pu wentru 'hombres', o la adición del sufijo - $k e$ al adjetivo en una frase nominal que cuente con este tipo de modificadores, como en küme-ke wentru 'hombres buenos'). No se establece concordancia, entendida esta como la manifestación de marcas morfológicas equivalentes y sistemáticas (Olate, Becerra y Alonqueo 2011; Olate, Pineda, Hasler y Soto, 2019). Con los determinantes ocurre lo mismo: mientras que para el español es obligatoria la flexión de las categorías mencionadas, en el mapudungun no lo es.

Las descripciones de las influencias que el mapudungun ejerce sobre el español mapuchizado sobre este punto alcanzan un relativo consenso 
entre los autores (Hernández y Ramos 1984, Lagos y Olivera 1988, Acuña y Menegotto 1996 y Contreras 2009). En el español de zonas de contacto, el número parece haber perdido la importancia que tiene en el español general: se conserva en el determinante, pero se pierde en el sustantivo, dejando de existir la concordancia entre ambos como se puede apreciar en los ejemplos (1)-(4). Junto con lo anterior, en ocasiones puede dejar de existir la concordancia entre el sujeto y su atributo, como se puede observar en (5).

(1) Ella muy contenta volve a los brazo de su mamá. (Acuña y Menegotto 1996: 258)

(2) Sin más mis lamento. (Acuña y Menegotto 1996: 258)

(3) Un pareja caballo (Hernández y Ramos 1984: 130)

(4) Lo mapuche no pregunta. (Lagos y Olivera 1988: 97)

(5) Hay machis que son buena y hay machis que son sucio. (Contreras 2009: 49)

Siguiendo a Acuña y Menegotto (1996), la estructura de la construcción nominal plural del español mapuchizado sería acorde con la del mapudungun, ya que presenta, típicamente, una sola marca de plural antepuesta al sustantivo en el determinante o el cuantificador $(C f$. Olate, Pineda, Hasler y Soto, 2019).

Con respecto al género, también se presentan numerosas discordancias entre el sustantivo y sus determinantes, las que también pueden extenderse a las oraciones de predicado nominal. Contreras (2009) señala que las diferencias se explican por la dificultad que representa la distinción de género para el hablante de las zonas de contacto, debido a que el mapudungun, como ya hemos señalado, no posee marcas morfemáticas para distinguir el género en las categorías nominales. Esto tiene como consecuencia que se generen enunciados como los siguientes, en que no se observa la concordancia de género característica del español general:

(6) Entonces tengo mucho cosa que contar. (Contreras 2009: 48)

(7) No estaba escaso la leña. (Contreras 2009: 49)

(8) Siéntate en la asiento del profesor. (Lagos y Olivera 1988: 97)

(9) Se forma un reunión. (Hernández y Ramos 1984: 130)

\subsection{LA ELISIÓN DE LA PREPOSICIÓN $A$ Y ORDEN DE LOS CONSTITUYENTES}

En el español hablado en zonas de contacto con el mapudungun se registra la omisión frecuente, pero no absoluta, de la preposición $a$, tanto en objetos 
directos humanos o animados, como en indirectos, en claro contraste con la norma del español general, en que dichas preposiciones se emplean: conozco a tu padre, le mostró el libro al niño. Los siguientes ejemplos ilustran el fenómeno. Los paréntesis explicitan la elisión de la preposición.

(10) Claro, () usted mi papá no lo conoce. (Contreras 2009: 50)

(11) Dejan () lo chanchito en la pajera. (Lagos y Olivera 1988: 99)

(12) La nena está mostrando el libro ( ) el nene. (Acuña y Menegotto 1996: 260)

Contreras (2009) propone que este fenómeno se produce debido a las diferencias entre los sistemas adposicionales de ambas lenguas: mientras el mapudungun solo tiene dos posposiciones mew y püle, el español tiene un sistema preposicional con un número mayor de componentes, por lo que "es explicable que el hablante mapuche al enfrentarse a un sistema preposicional más complejo, como es el del castellano, manifieste inseguridad" (Contreras 2009: 50). Esta interpretación es compartida por Lagos y Olivera (1988). Finalmente, Acuña y Menegotto (1996) agregan que la falta de la preposición $a$, en los casos en que participan terceras personas agente y paciente animados, produce ambigüedad para un hablante de español estándar. Sin embargo, proponen que en el español mapuchizado el sistema se ha reinterpretado, pues junto con la posición canónica VO propia del español, este adquiere el requisito de adyacencia de los complementos, propio de la lengua mapuche, razón por la cual el objeto indirecto pasa a colocarse obligatoriamente después del objeto directo en estos casos. Por ejemplo:

(13) Le rompió lo dibujo la nena un nene. (Acuña y Menegotto 1996: 261)

Como es fácil advertir, para un hablante de español general es difícil discernir la estructura argumental de este ejemplo; sin embargo, en esta variedad de español mapuchizado la ambigüedad se resuelve pues el objeto indirecto va siempre después del objeto directo (lo dibujo, en este caso). Por esta razón, en (13) la nena corresponde al objeto indirecto y un nene al sujeto de la oración, es decir: un nene le rompió el dibujo a la nena.

\subsection{USO DIFERENCIAL DEL PRONOMBRE ÁTONO EN LOS VERBOS PRONOMINALES}

En la variedad del español en contacto, es posible encontrar oraciones en las que los pronombres átonos de los verbos pronominales resultan omitidos, como se puede observar en los siguientes ejemplos: 
(14) (me) levanto po y (me) lavo la cara. (Lagos y Olivera 1988:97)

(15) Y ahora portó mal pu. (Hernández y Ramos 1984:132)

Con respecto a este fenómeno, se presentan diversas explicaciones. Por un lado, Lagos y Olivera (1988) plantean que el complejo sistema de pronombres del español que posee distinciones de persona, género, número y caso resulta un serio problema para el hablante de mapudungun, ya que el mapudungun señala dichas distinciones mediante el sintagma verbal, a partir del sistema de personas satélites (Salas [1992] 2006); por esta razón, se omiten los pronombres átonos o se usan de manera inapropiada, sobre utilizándose, confundiéndose entre sí o con los pronombres tónicos. Con respecto a este fenómeno, Acuña y Menegotto (1996) señalan que la elisión de los pronombres se produce porque en el mapudungun existe la posibilidad de omitir el pronombre acusativo si el referente ya fue mencionado en el discurso. A nuestro juicio, ambos factores se complementan entre sí y juegan un rol importante en la explicación de este uso diferencial.

\subsection{EXPRESIÓN DEL OBJETO EN TERCERA PERSONA}

En Olate, Wittig y Hasler (2014), se reportan los siguientes casos en producciones de niños monolingües en castellano que viven en un contexto de bilingüismo social mapudungun-castellano:

(16) "Después el niñito lo tiran al río con el perro"

(17) "y empezaron a llamarlo (a la rana)"

(18) "Aquí el niño andaba en la ventana llamándolo (a la rana)"

(19) "Y después el niñito lo empezó a llamar por un tubo de una ardillita (a la rana)"

(20) "Después el animal lo botó en una laguna y el perrito también (al niño y al perro)"

(21) “y después el niño despertó y no lo vio nada (a la rana). Y lo buscaba por todas partes (a la rana)"

(22) "lo anda picando las abejas (al perro)"

En estos casos, se observa la predominancia del pronombre lo, que se entiende como una transferencia del mapudungun al español, provocando la reestructuración del sistema de pronombres átonos. Desde el plano de los argumentos centrales del verbo, el mapudungun es una lengua de marcación en el núcleo (head-marking) (Golluscio 2010). En contraste, el español es una lengua de marcación en los dependientes (dependent-marking), en 
transición hacia una de marcación en el núcleo (Belloro 2004). En el caso del objeto de tercera persona, en mapudungun este es realizado con el sufijo $-f i$, en construcciones directas, y la forma discontinúa -e-y-ew, en inversas y se transfiere al español a partir de la reestructuración del sistema de pronombres átonos, observada principalmente en el surgimiento del clítico lo invariable, que pasa a funcionar de manera análoga a la indexación de argumentos centrales típica de las lenguas de marcación en el núcleo (Olate, Wittig y Hasler 2014).

Desde una gramática de la referencia (Givón 2005), la indexación del tópico secundario presenta equivalencias y diferencias en las lenguas en cuestión. Si bien ambas tienen un recurso especializado en marcar o indexar el elemento que sintácticamente se interpreta como objeto directo, la diferencia radica en la naturaleza del sistema de clíticos del español, y más específicamente, en los pronombres de tercera persona, que están regidos por la concordancia nominal de género, número y caso. En contraste, el mapudungun posee sufijos pronominales que marcan en el verbo los diferentes tipos de tópico (Olate, Wittig y Hasler 2014).

\section{DISCURSO: GRAMÁTICA Y PRAGMÁTICA}

\subsection{DISCURSO REFERIDO Y EVIDENCIALIDAD}

En Hasler (2012) y Hasler y Soto (2016), se señala que el discurso referido del mapudungun puede ser expresado por una cláusula independiente con un predicado de decir y una cláusula subordinada nominalizada que puede expresar su sujeto a través del pronombre posesivo correspondiente y tomar cualquier operador clausal, como se puede observar en el siguiente ejemplo:
(23) feypi-n
ta-mi
chillkatu-a-el
Buenos Aires mew
decir-IND.1SG
DET1-2SG.POS estudiar-FUT-NMR2
Buenos Aires PPOS
'Yo dije que estudiarás en Buenos Aires.'

Sin embargo, esta construcción tiene una frecuencia muy baja; en general, en mapudungun se prefiere el discurso directo (24), el sufijo evidencial -rke (25) o el marcador discursivo piam (26) para expresar este tipo de significado:

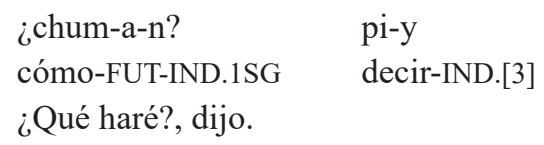


(25) amu-rke-ymi mi

pe-me-a-fi-el

Maria

ir-EVID-IND.2SG 2SG.POS

ver-DIR3-FUT-3.OP-NMR2

Maria

Dicen que fuiste a ver a María

(26) $\mathrm{Pu}$ Catrüan che piam ye-y=engün kom kiñewün-küle-ke-fu-y

PL Catrian gente EVID.REP llevar-IND.[3]=PL todo unido-EST-HAB-AP-IND.[3]

kom tüfa ta Dollümko che, doy kuyfi ta-ti.

todo DEM3 DET2 Dollümko gente más antes DET2-DET1

Los Catrian estaban unidos con los Dollümko, dicen. Eso era antiguamente.

(Relmuan 1997: 23)

El sufijo -rke y el marcador piam funcionan como operadores con un significado reportativo, aunque no citativo, pues este último se expresa principalmente por el discurso directo. Esto significa que cuando se expresa la fuente del discurso referido, se emplea el discurso directo sin cambiar las coordenadas deícticas del discurso original, mientras que cuando la fuente es desconocida o irrelevante, los hablantes de mapudungun usan los marcadores evidenciales, situando las coordenadas deícticas en el hablante.

Considerando lo anterior, en Hasler (2012) y Hasler y Soto (2016) se propone que el dominio evidencial del mapudungun se transfiere al español a partir de la reestructuración general del sistema de discurso referido, producido por la gramaticalización de la construcción de discurso indirecto, que se transforma en el marcador evidencial reportativo dicen que (dicen en posición final). En otras palabras, un sistema que ya existía en el español se reinterpreta para expresar una categoría gramatical del mapudungun.

En particular, esta reestructuración tiene lugar a partir de tres cambios inducidos por contacto: a) gramaticalización del verbo decir; b) gramaticalización del subordinador que en construcciones de discurso indirecto que toman un discurso directo como unidad dependiente y c) empaquetamiento del discurso directo en otras cláusulas de discurso directo.

\subsection{GRAMATICALIZACIÓN DEL VERBO DECIR}

El verbo decir, en contextos de discurso indirecto, se fija en tercera persona, deja de expresar el predicado principal de la construcción completiva y pasa a ser usado como marcador evidencial. Por ejemplo:

(27) ya po y dice que un hombre iba a bañar al río, un joven y siempre se encontraba con una señorita linda, bonita y ese joven se acostumbró tanto, y dice que al otro día pusieron de acuerdo po. 
En Hasler (2012) se plantea un contraste de la frecuencia y el significado del discurso indirecto en español hablado en Santiago y en zonas de contacto, cuyos datos adjuntamos en el Cuadro 2, donde se observa que en este último caso el discurso indirecto ocurre solamente con valor evidencial. Para realizar este análisis sobre el español en contacto con el mapudungun, se entrevistó a 9 sujetos de la comunidad de Curaco Ranquil (IX Región), con distintos niveles de bilingüismo mapudungun-español, lo que generó un corpus de 8 horas y 45 minutos, que fue contrastado con una muestra del corpus PRESEEA-SA, compuesta por 9 entrevistas semiestructuradas realizadas a hablantes de Santiago de Chile, de aproximadamente una hora de duración cada una. Los resultados fueron los siguientes:

\begin{tabular}{|c|c|c|c|c|}
\hline \multirow{4}{*}{$\begin{array}{l}\text { Español de Chile } \\
\text { Grupo 1: Hablantes bi- } \\
\text { lingües subordinados de } \\
\text { español, con un apren- } \\
\text { dizaje tardío de esta úl- } \\
\text { tima lengua y con bajo } \\
\text { nivel de intensidad de } \\
\text { interacciones con otras } \\
\text { variedades de ella. }\end{array}$} & \multicolumn{2}{|c|}{$\begin{array}{l}\text { DI con valor evi- } \\
\text { dencial }\end{array}$} & \multirow{2}{*}{$\begin{array}{l}\text { Otros usos } \\
\text { de DI } \\
25(68 \%) \\
\end{array}$} & \multirow{2}{*}{\begin{tabular}{|l|} 
Total \\
37 \\
95
\end{tabular}} \\
\hline & \multicolumn{2}{|c|}{$12(32 \%)$} & & \\
\hline & $\begin{array}{c}\text { De } \\
\text { oídas }\end{array}$ & Citativo & 0 & 95 \\
\hline & 95 & 0 & & \\
\hline $\begin{array}{l}\text { Grupo 2: Hablantes bi- } \\
\text { lingües coordinados de } \\
\text { transición, bilingües } \\
\text { tempranos y/o que inte- } \\
\text { ractúan frecuentemente } \\
\text { con otras variedades del } \\
\text { español }\end{array}$ & 26 & 12 & 12 & 50 \\
\hline $\begin{array}{l}\text { Grupo 3: Hablantes mo- } \\
\text { nolingües de español } \\
\text { que habitan en la comu- } \\
\text { nidad }\end{array}$ & 16 & 9 & 0 & 27 \\
\hline
\end{tabular}

Cuadro 2: Frecuencia y significado del discurso indirecto en español y en español mapuchizado (tomado de Hasler 2012). 


\subsection{GRAMATICALIZACIÓN DEL SUBORDINADOR 'QUE' EN CONSTRUCCIONES DE DISCURSO INDIRECTO QUE TOMAN UN DISCURSO DIRECTO COMO UNIDAD DEPENDIENTE}

La gramaticalización del subordinador que es, probablemente, resultado de la combinación recurrente del discurso directo y el operador evidencial en mapudungun. En estos casos, que ya no funciona como subordinador y, de hecho, no se producen cambios en los centros deícticos involucrados. Desde un punto de vista semántico, que se usa, en este contexto, como marcador evidencial. Por ejemplo:

(28)Ya po y le sirvieron: "aquí tiene porotos mamita, sírvase". "No, este poroto yo no como" que dijo. "Ta la lesera oh". Ya po, y no comió no más po. "No, este poroto no" que dijo. Ya y, y era locro que comía po este

\subsection{EMPAQUETAMIENTO DEL DISCURSO DIRECTO EN OTRAS CLÁUSULAS DE DISCURSO DIRECTO}

Dado que el discurso indirecto casi no se emplea en mapudungun, el discurso directo se usa para expresar todo el campo del discurso referido. Como consecuencia de esto, es posible encontrar construcciones de discurso directo dentro de otras construcciones de discurso directo. Por ejemplo:

(29)Y dice después la abuelita: "fue él entró corriendo a la casa y me jue a tomar de la mano y me trajo a la rastra", y yo le dije: "qué te pasa chacha", le dije, "ay es que D va a tener la guagua" me dijo cuando veníamos, me traía a la rastra "a pasear", me dijo, dijo. "No me puse ni delantal limpio mamita" me dijo cuando llegó.

\section{CONCLUSIONES}

El presente trabajo no tuvo como objetivo volver a discutir, en los términos científicos e ideológicos propios de la primera mitad del siglo pasado, la tesis sustratista, o indigenista, propuesta por Lenz, sino mostrar la persistencia de sus intuiciones, conceptos y metodologías en la lingüística de contacto actual. Para ello, revisamos una serie de fenómenos de distintos niveles en 
que puede apreciarse la utilidad de planteamientos y estudios desarrollados por Lenz. Tras años de cierto olvido o minusvaloración en la lingüística hispánica, tendencias actuales de la contactología nos permiten apreciar a un Lenz visionario que, anticipándose a su época y contra el clima ideológico vigente en su entorno hispanoamericano, sentó las bases para el estudio científico del español en contacto con el mapudungun y, más ampliamente, en contacto con lenguas indígenas.

La convicción de Lenz lo llevó a desarrollar reflexiones previas con el objeto de abordar la situación de contacto mapudungun-castellano. Entre ellas destaca la idea de que el conocimiento estructural de las lenguas en contacto es un requisito fundamental para pesquisar este fenómeno (Thomason y Kaufman 1988; Ansaldo 2004; Heine y Kuteva 2005; Matras 2009; Aikhenvald y Dixon 2006, entre otros):

Empeñado en el estudio del desarrollo especial que el castellano ha sufrido en boca del pueblo bajo de Chile, tenía que procurarme conocimientos de la lengua indígena para averiguar si acaso este desarrollo se debía en parte a influencias ejercidas por el idioma de los aboríjenes del país (Lenz 1895-1897: IV).

En cuanto a la dimensión tipológica del contacto, Lenz, advirtiendo que sus conocimientos de filología románica no son suficientes para un estudio detallado de la relación entre el castellano y la lengua mapuche, utiliza sus conocimientos en filología comparada de lenguas indoeuropeas para iniciar la indagatoria. Sin embargo, con la perspicacia que lo caracteriza, pronto se da cuenta de que las gramáticas modernas están muy influidas por la gramática latina, por lo que prevé la dificultad para construir una gramática descriptiva de la lengua indígena. En cuanto al método, afirma que seguirá la propuesta de von der Gabelentz, verdadero padre de la tipología. Su intuición tipológica y de contacto se observa en el siguiente párrafo:

Me parece que el análisis lójico i sicolójico de la sintáxis de idiomas que, en comparación con el mecanismo inextricable de las lenguas indo-europeas, son relativamente diáfanos i sencillos ha de ayudarnos mucho para revelar los misterios del pensamiento i la jénesis del habla humana. Solo la comparación con idiomas de índole completamente distinta nos puede enseñar las particularidades de nuestra lengua. (Lenz 1895-1897: IV)

Otro aspecto que permite observar la vigencia del trabajo de Lenz tiene que ver con la mirada ecológica del proceso de mestizaje lingüístico-cultural que intentó describir. En la actualidad, una gran cantidad de trabajos ponen como punto de partida la ecolingüística o el entorno sociocultural, histórico, 
demográfico, geográfico y político de las lenguas en contacto (Johanson 2002a; Mufwene 2001; Godenzzi 2007; Ansaldo 2004; entre otros). Esta perspectiva comprehensiva puede apreciarse en la siguiente cita:

Trasplantada a los vastos territorios de la América la lengua de los conquistadores castellanos, tuvo que amoldarse a la naturaleza antes desconocida del nuevo mundo, tuvo que adaptarse a otro sistema de vida. (Lenz 1912: 4)

Esta idea es similar a la consignada en Godenzzi (2007), que muestra los procesos de universalización, vernacularización y emergencia que tiene el castellano en su proceso de desarrollo histórico en América. Junto a lo anterior, es necesario observar que la tesis sustratista de Lenz se propone en un contexto particular, el chileno, en que la sociedad indígena que usaba la lengua de sustrato estaba en pleno proceso de anexión por parte de un Estadonación. Este hecho debe consignarse en el contexto de lo que Zimmermann (2001) entiende como las relaciones del español americano con sustratos indoamericanos vivos que aún gozan de hablantes y funcionalidad.

Amparados en el principio lenziano de comparación de lenguas como llave para la comprensión del castellano, podemos advertir, por último, de que, indirectamente, un aporte fundamental del lingüista alemán fue la intuición de que el castellano y las lenguas en general tienen dominios sensibles al cambio y que cuando estas entran en interacción, generan modificaciones genéricas o particulares que son movilizadas por los hablantes.

\section{REFERENCIAS BIBLOGRÁFICAS}

ACUÑa, LeONOR y ANDREa MENEGOTto. 1996. El contacto lingüístico español mapuche en la Argentina. Signo y Seña 6: 235-271.

Aikhenvald, Alexandra y Robert Dixon. 2007. Grammars in contact: a cross-linguistic perspective. En Alexandra Aikhenwald y Robert Dixon (eds). Grammars in Contact: ad Cross-linguistic Typology, pp. 1-66. Oxford: Oxford University Press.

Alonso, Amado. 1925. El grupo 'tr' en España y América. Homenaje ofrecido a Menéndez Pidal: Miscelánea de estudios lingüísticos, literarios e históricos, Vol. II, pp. 167-191. Madrid: Librería y casa editorial Hernando.

Alonso, Amado. 1940a. Rodolfo Lenz y la dialectología hispanoamericana. En VV.AA., El español en Chile. Trabajos de Rodolfo Lenz, Andrés Bello y Rodolfo Oroz. Traducción, notas y apéndices de Amado Alonso y Raimundo Lida. Buenos Aires: Biblioteca de Dialectología Hispanoamericana.

Alonso, Amado. 1940b. La interpretación araucana de Lenz para la pronunciación chilena. En VV.AA. El español en Chile. Trabajos de Rodolfo Lenz, Andrés Bello y Rodolfo 
Oroz. Traducción, notas y apéndices de Amado Alonso y Raimundo Lida. Buenos Aires: Biblioteca de Dialectología Hispanoamericana.

Ansaldo, Umberto. 2004. Contact, typology and the speaker: the essentials of language. Languages Sciences 26: 485-494.

Belloro, Valeria. 2004. A Role and Reference Grammar account of third-person clitic clusters in Spanish. Tesis para optar al grado de Master of Arts, State University of New York at Buffalo.

Bernaschina, Vicente. 2013. Rodolfo Lenz, 150 años en disputa con el imperio de la lengua. Universum 28 (2): 117-138.

CANField, D. Lincoln. 1988. El español de América. Barcelona: Crítica. [Primera edición en inglés, Spanish pronunciation in the Americas. Chicago: University of Chicago Press, 1981].

Contreras, Constantino. 1998. Oyente de dos lenguas, hablante de una: situación actual del escolar mapuche. Lingüística Española Actual 20 (2): 269-290.

Contreras, Constantino. 1999. El castellano hablado por mapuches. Rasgos del nivel morfosintáctico. Estudios Filológicos 34: 83-98.

Contreras, Constantino. 2009. El castellano hablado en un área de contactos. Boletín de Filología 44 (2):39-63.

Febrés, ANDRÉs. 1765. Arte de la Lengua General del Reyno de Chile, con un diálogo chilenohispano muy curioso.... Lima.

Fontanella de Weinberg, María Beatriz. 1992. El español de América. Madrid: Mapfre. Giese, Wilhelm. 1947-1949. Hispanismos en el mapuche. Boletín de Filología 5: 115-132.

Givón, TALmy. 2005. Context as other minds: The pragmatics of sociality, cognition and communication. Amsterdam/Philadelphia: John Benjamins Publishing.

Godenzzi, Juan Carlos. 2007. El español de América y el español de los Andes: universalización, vernacularización y emergencia. En Martina Schrader-Kniffki y Laura Morgenthaler (eds.). La Romania en interacción: entre contacto, historia y politica. Ensayos en homenaje a Klaus Zimmermann, pp. 29-50. Frankfurt: VervuertIberoamericana.

Golluscio, Lucía. 2010. Ditransitives in Mapudungun. En Andrej Malchukov, Martin Haspelmath y Bernard Comrie (eds.). Studies in Ditransitive Constructions. A Comparative Handbook, pp. 711-756. Berlín: De Gruyter Mouton.

Hasler, FeliPe. 2012. El sistema de evidencialidad en mapudungun y sus transferencias al español mapuchizado. Tesis para optar al grado de Magíster en Lingüística con mención en lengua española, Universidad de Chile.

Hasler, Felipe y Guillermo Soto, 2012. Determinación de algunas propiedades del subsistema consonántico del mapudungun y el del español de Chile en los siglos XVI y XVII a partir de los hispanismos léxicos. En Hebe González y Beatriz Gualdieri (eds.). Lenguas Indigenas de América del Sur I: Fonología y Léxico, pp. 91-102. Mendoza: Editorial FFyL-UNCuyo y SAL.

Hasler, FeliPe y Guillermo Soto. 2016. The influence on Spanish of reported speech in Mapudungun complex syntax and language contact. Ponencia presentada en el congreso VII Syntax of the World's Languages. Universidad Nacional Autónoma de México, Ciudad de México, México.

Heine, Bernd y TANiA Kuteva. 2005. Language contact and grammatical change. Cambridge: Cambridge University Press.

Hernández, Arturo y Nelly Ramos. 1984. Algunas características gramaticales del castellano hablado por mapuches. Actas. Jornadas de Lengua y Literatura mapuche 1: $33-45$. 
JOHANSON, LARS. 2002a. Contact-induced linguistic change in a code-copying framework. En Mari C. Jones y Edith Esch (eds.). Language Change: The Interplay of Internal, External and Extra-linguistic Factors, pp. 285-313. Berlin: Mouton de Gruyter.

JOHANSON, LARS. 2002b. Do languages die of structuritis? On the role of code-copying in language endangerment. Rivista di Linguistica 14(2): 249-270.

Lagos, Daniel y Selma Olivera. 1988. Algunas características del español hablado por los escolares mapuches de la comuna de Victoria. Estudios Filológicos 23: 89-102.

LAPESA, RAFAEL. 1991. El estudio del español en los últimos decenios: aportaciones y cuestiones pendientes. En César Hernández, Germán de Granda, Carmen Hoyos, Vicenta Fernández, Deborah Anne Dietrick y Yolanda Carballera (eds.). El español de América: actas del III congreso internacional de el español en América, pp. 7-27. Valladolid: Junta de Castilla y León, Pabecal.

LAPESA, RAFAel. 1992. Nuestra lengua en España y América. Revista de Filología Española 72 (3-4): 269-282.

Lenz, Rodolfo. 1894. Ensayos filolójicos americanos. Anales de la Universidad de Chile LXXXVII: $113-132$ y 353-367.

LenZ, Rodolfo. 1895-1897. Estudios Araucanos. Materiales para el estudio de la lengua, la literatura i las costumbres de los indios mapuche o araucanos. Santiago: Cervantes.

Lenz, Rodolfo. 1912. Los elementos indios del castellano de Chile. Actas del XVII Congreso Internacional de Americanistas. Buenos Aires.

Lenz, Rodolfo. 1940. Estudios chilenos (Fonética del castellano de Chile). En VV.AA., El español en Chile. Trabajos de Rodolfo Lenz, Andrés Bello y Rodolfo Oroz. Traducción, notas y apéndices de Amado Alonso y Raimundo Lida. Buenos Aires: Biblioteca de Dialectología Hispanoamericana. [Traducción corregida de «Chilensiche Studien, I-VII», 1892-1893].

Lope Blanch, Juan Manuel. 1992. La falsa imagen del español americano. Revista de Filología Española (3-4): 313-336.

Matras, Yaron. 2009. Language contact. Cambridge: Cambridge University Press.

Matras, Yaron. 2013. Contact, convergence, and tipology. En Raymond Hickey (ed.). The handbook of language contact, pp. 66-85. Oxford: Wiley-Blackwell.

Mufwene, Salikoko. 2001. The ecology of language evolution. Cambridge: Cambridge University Press.

Olate, Aldo; Rodrigo Becerra y Paula Alonqueo. 2011. Cambio lingüístico y contacto de lenguas. Nuevas miradas e interpretaciones en torno al castellano de América. Lenguas Modernas 38: 37-62.

Olate, Aldo; Fernando Wittig y Felipe Hasler. 2014. Análisis tipológico-funcional de un rasgo del español de contacto mapuche/castellano. Onomázein 30: 169-189.

Olate, Aldo. 2017. Contacto lingüístico mapuzugun/castellano. Aspectos históricos, sociales y lingüísticos. Revisión bibliográfica y propuesta de análisis desde la dimensión morfosintáctica y tipológica. Onomázein 36: 122-158.

Olate, Aldo; Ricerdo Pineda; Felipe Hasler Felipe y Guillermo Soto. (2019). “Y después ya cambió eso cuando aprendió a hablar wingka los mapuche. La concordancia de número en el castellano hablado por bilingües mapuzugun-castellano". Círculo de Lingüística Aplicada a la Comunicación, 78: 211-232.

Oroz, Rodolfo. 1962. La lengua castellana en Chile. Santiago: Universidad de Chile.

Palacios, Azucena. 2011. Nuevas perspectivas en el estudio del cambio inducido por contacto: Hacia un modelo dinámico del contacto de lenguas. Lenguas Modernas 38: 17-36.

Penny, Ralph. 2000. Variation and change in Spanish. Cambridge: Cambridge University Press. 
Relmuan, María. 1997. Kiñeke nütram ka pentukun dungu feypiel pu Rapawe ka Rukapangui lof che. Temuco: UFRO.

SAlas, AdAlBerto. [1992] 2006. El mapuche o araucano. Fonología, gramática y antología de cuentos. Edición de Fernando Zúñiga. Santiago: Centro de Estudios Públicos.

SÁnchez MÉndez, JuAn. 2003. Historia de la lengua española en América. Valencia: Tirant lo Blanch.

SÁnCHEZ, GilberTo. 1992. La contribución del Dr. Rodolfo Lenz al conocimiento de la lengua y cultura mapuches. Boletín de Filología 33: 273-299.

SAPIR, EDWARD. 1921. Language. An introduction to the study of speech. New York: Harcourt, Brace.

Siemund, Peter y Noemí Kintan (eds.). 2008. Language contact and contact languages (Vol. 7). Amsterdam/Philadelphia: John Benjamins Publishing.

Soto, Guillermo y Felipe Hasler. 2011. Introducción: Lenguaje, cognición y cultura. Nuevas perspectivas sobre el contacto lingüístico. Lenguas Modernas 38: 9-16.

Thomason, Sara. 2001. Language contact. An introduction. Washington: Georgetown University Press.

ThOMASON, SARA. 2013. Contact explanations in linguistics. En Raymond Hickey (ed.). The handbook of language contact, pp. 31-47. Oxford: Wiley-Blackwel.

Thomason, Sara y Terence Kaufman. 1988. Language contact, creolization, and genetic linguistics. Los Angeles: University of California Press.

ZimmermanN, Klaus. 2001. Interculturalidad y contacto de lenguas: condiciones de la influencia mutua de las lenguas amerindias con el español. En Klaus Zimmermann y Thomas Stolz (eds.). Lo propio y lo ajeno en las lenguas austronésicas y amerindias. Procesos interculturales en el contacto de lenguas indígenas con el español en el Pacifico e Hispanoamérica. Madrid: Vervuert- Iberoamericana. 